BNL-112541-2016-JA

\title{
Supercontinuum Fourier transform spectrometry with balanced detection on a single photodiode
}

\author{
Vasily V. Goncharov and Gregory E. Hall
}

Submitted to Journal of Chemical Physics

August 2016

Chemistry Department

Brookhaven National Laboratory

\author{
U.S. Department of Energy \\ USDOE Office of Science (SC), \\ Basic Energy Sciences (BES) (SC-22)
}

Notice: This manuscript has been authored by employees of Brookhaven Science Associates, LLC under Contract No. DE- SC0012704 with the U.S. Department of Energy. The publisher by accepting the manuscript for publication acknowledges that the United States Government retains a non-exclusive, paid-up, irrevocable, world-wide license to publish or reproduce the published form of this manuscript, or allow others to do so, for United States Government purposes. 


\section{DISCLAIMER}

This report was prepared as an account of work sponsored by an agency of the United States Government. Neither the United States Government nor any agency thereof, nor any of their employees, nor any of their contractors, subcontractors, or their employees, makes any warranty, express or implied, or assumes any legal liability or responsibility for the accuracy, completeness, or any third party's use or the results of such use of any information, apparatus, product, or process disclosed, or represents that its use would not infringe privately owned rights. Reference herein to any specific commercial product, process, or service by trade name, trademark, manufacturer, or otherwise, does not necessarily constitute or imply its endorsement, recommendation, or favoring by the United States Government or any agency thereof or its contractors or subcontractors. The views and opinions of authors expressed herein do not necessarily state or reflect those of the United States Government or any agency thereof. 


\title{
Supercontinuum Fourier transform spectrometry with balanced detection on a single photodiode
}

\author{
Vasily V. Goncharov a and Gregory E. Hall* \\ Division of Chemistry, Department of Energy and Photon Sciences \\ Brookhaven National Laboratory, Upton, NY 11973, USA
}

(Received 10 June 2016; Revised 11 August 2016; Accepted 13 August 2016)

a present address: Janelia Farm Research Campus, Howard Hughes Medical Institute, Ashburn, VA 20147 USA

*corresponding author gehall@bnl.gov (631)344-4376 
We demonstrate a method of combining a supercontinuum light source with a commercial Fourier transform spectrometer, using a novel approach to dual-beam balanced detection, implemented with phase-sensitive detection on a single light detector. A $40 \mathrm{~dB}$ reduction in the relative intensity noise is achieved for broad-band light, analogous to conventional balanced detection methods using two matched photodetectors. Unlike conventional balanced detection, however, this method exploits the time structure of the broadband source to interleave signal and reference pulse trains in the time domain, recording the broadband differential signal at the fundamental pulse repetition frequency of the supercontinuum. The method is capable of real-time correction for instability in the supercontinuum spectral structure over a broad range of wavelengths, and is compatible with commercially designed spectrometers. A proof-ofprinciple experimental setup is demonstrated for weak absorption in the 1500-1600 nm region.

PACS codes: 07.57.-c; 33.20.Ea; 82.80.Dx 


\section{INTRODUCTION}

The broadband optical pulse trains generated from mode-locked lasers in highly nonlinear optical fibers, known as supercontinua, have found numerous applications in metrology and spectroscopy ${ }^{1-3}$ The spatial coherence, brightness, and ease of focusing encourage their use for a variety of illumination and spectroscopic applications. When used directly as a source for absorption spectroscopy, ${ }^{4,5}$ these advantages are tempered by some disadvantages to be overcome ${ }^{6}$ related to fluctuations, both in the total intensity as well as variations in spectral content. Some of the fluctuations are intrinsic to the highly nonlinear processes, seeded by quantum noise, that generate the supercontinuum, ${ }^{7}$ while additional excess noise in supercontinuum sources is common and related to the amplification of technical noise from the pump source.

Fluctuation noise is particularly pernicious in Fourier Transform Spectrometry (FTS), leading to a multiplex disadvantage, ${ }^{8,9}$ where multiplicative source noise appears on all resolved spectral detection channels simultaneously, in contrast to additive detector noise that is effectively divided among all detection channels. For this reason, minimizing or correcting for source fluctuations should be given high priority. Dual beam, differential measurements are standard procedure in direct absorption dispersive instrumentation, often implemented with a pair of matched light detectors. ${ }^{10}$ Numerous applications of differential detection have been developed for FTS, ${ }^{4,9,11-18}$ usually implemented in a Michelson interferometer using the complementary balanced and unbalanced (forward and backward) beams. The optical or digital processing of these two interferometer beams differs from conventional dual beam differential spectroscopy in that source 
fluctuations appear in phase on both beams, whereas the interferometric modulations are out of phase. Assorted applications have been described using dual-beam FTS with optical superposition of the two beams on a single detector, ${ }^{12-14}$ as well as electronic differential processing of the two beams with separate matched detectors ${ }^{4}$, including designs based on electronic autobalancing detectors. ${ }^{17-20}$ We have recently demonstrated $^{21}$ balanced FTIR using an external broadband polarizing beamsplitter to divide the forward, modulated output of a commercial FTIR spectrometer into signal and reference beams. The optical source was a mode-locked near-infrared fiber laser and matched InGaAs detectors simultaneously measured the difference interferogram and a single-channel reference interferogram. The signal processing then resembles that developed for the sum and difference measurements recorded in polarization modulation FTIR. $^{22,23}$

The present communication describes and demonstrates a simplification and improvement on our previous work, ${ }^{21}$ in which a single photodiode is used to implement balanced detection, by temporal interleaving of sample and reference beams, recording the difference interferogram with phase-sensitive detection at the mode-locked laser pulse repetition rate, and the sum interferogram after low-pass filtering to average the signals from sample and reference beams. The method has been demonstrated with a spectrally filtered portion of a supercontinuum, achieving strong enough suppression of the source noise to detect weak single-pass absorption spectra close to the shot noise limit. This method is a good match for sensitive measurement of weak signals such as those encountered in surface reflection/attenuation methods, where the baseline stability and 
reproducibility limits the detectable signals, and when aggressive pathlength multiplication by multipass or cavity methods ${ }^{17,18}$ are less practical than for gas or liquid samples.

As with our previous work, no permanent modifications are required to a commercial FTIR spectrometer from which only the forward beam is accessible. The method uses a synchronous two-channel acquisition of interferograms, sharing many features of the sum and difference data acquisition and signal processing schemes developed for polarization modulation FTIR methods ${ }^{22-24}$ in circular dichroism and attenuated reflection FTIR spectroscopy.

\section{EXPERIMENTAL}

\section{Optical Design}

The optical layout implemented for dual-beam FTIR spectroscopy is illustrated in Figure 1 , resembling apparatus we have previously described, ${ }^{21}$ but with a modified source and detection scheme. In the present work, the source is a bandpass-filtered spectral region of a supercontinuum, generated in a photonic crystal fiber pumped by an amplified, homebuilt, mode-locked Er fiber laser with a repetition rate of $\sim 100 \mathrm{MHz}$. The full spectral range of the supercontinuum source is roughly $1100-2300 \mathrm{~nm}$, and the relative intensity noise (RIN) was measured to be around $-110 \mathrm{dBc} / \mathrm{Hz}$. The FTIR spectrometer is a Bruker Vertex 80V, fitted with flanges containing optical fiber feed-through connections to introduce the source and extract the modulated beam. Fiber collimators on translation stages installed inside the spectrometer housing could be moved in and out of the interferometer beam path under computer control, without disturbing the alignment or the conventional operation of the built-in incoherent sources or detectors. After leaving the 
interferometer, the beam is returned to free space propagation and split into balanced signal and reference arms, employing broadband optical polarization components. A stack of long- and short-pass optical filters restricts the spectral content to the operational range of the polarizing optics $(1200-1650 \mathrm{~nm})$ or a more restrictive region of interest. The first polarizer cleans up the polarization state of the modulated and bandpass-filtered supercontinuum output, while the waveplate and second clean-up polarizer are adjusted to balance the average optical power in the two arms, having less dependence on ambient temperature and optical power than the waveplate alone. In this implementation, the combination of the broadband waveplate and polarizing beamsplitter provided at least 70 $\mathrm{dB}$ of common-mode RF power rejection, measured at the $100 \mathrm{MHz}$ repetition rate of the supercontinuum source, while maintaining identical spectral content between both signal and reference arms to within $0.3 \%$ in the operational range between 1200 and $1650 \mathrm{~nm}$. An optical delay of $\sim 5 \mathrm{~ns}\left(180^{\circ}\right.$ phase shift at $\left.100 \mathrm{MHz}\right)$ was introduced into the reference beam path prior to recombining the orthogonally polarized signal and reference beams in a second polarizing beam splitter. In this way, interleaved even and odd numbered pulses arriving at twice the repetition rate of the supercontinuum source constitute collinear signal and reference beams that can be focused onto a single photodetector. The InGaAs detector (New Focus 1611-AC) provides separately amplified DC $(<20 \mathrm{kHz})$ and RF $(20 \mathrm{kHz}-1 \mathrm{GHz})$ signals, respectively sampling the average amplitudes of the two beams, and the alternating signal and reference pulse amplitudes. The total optical power in the combined beam was $\sim 400 \mu \mathrm{W}$. To avoid distortion of the DC channel inteferogram, the mirror speed was limited to keep the $\mathrm{HeNe}$ fringe rate $\leq 10 \mathrm{kHz}$. Phase-sensitive detection of the AC-coupled signal at the $100 \mathrm{MHz}$ 
pulse repetition rate isolates the nulled interferogram, broken only by the differential transmission of the two beams due to the small $(0.3 \%)$ and reproducible spectral mismatch of optical components, and the desired sample absorption in the signal arm. The local oscillator for the mixer is derived from a photodiode viewing the optical pulse train of the source, with a phase adjusted by optimizing the mixer signal while the reference beam is blocked. The optical DC balance is adjusted with the waveplate and polarizer upstream from the polarizing beamsplitter, alternately blocking each of the two arms. The RF balance is then optimized by fine adjustment of the optical delay between signal and reference arms with an empty sample cell. The 24-bit digitizers in the Bruker interferometer simultaneously acquire two interferograms: one from the DC coupled channel, proportional to the sum of reference and signal, the other from the RF channel, proportional to the difference of signal and reference, after phase sensitive detection and low pass filtering. The differential interferogram has strongly suppressed common-mode RIN, but since its amplitude is deliberately minimized, identification of the zero path difference and phase corrections require additional care, as described below. When combined with the simultaneously acquired sum interferogram, however, algebraic signal processing can provide the desired result of a low-noise absorption spectrum, corrected for source fluctuation and drift.

\section{Signal processing}

In addition to the HeNe fringe sampling, two synchronous and parallel, 24-bit resolution analog-to-digital converter (ADC) channels are provided in the Bruker Vertex interferometers, used in a similar manner for simultaneous acquisition of sum and difference interferograms in polarization modulation spectroscopy. ${ }^{22-24}$ The goal of the 
signal processing scheme is to take advantage of the enhanced amplitude resolution and suppressed common-mode noise of the differential interferogram to generate algebraically a pair of interferograms representing the signal and reference arms which, when Fourier transformed into signal and reference spectra, have a ratio that is dominated by the weak signal absorption and is nearly independent of the fluctuations in the source intensity or spectral content. Phase correction of differential signals presents well-known challenges ${ }^{25}$ since the amplitude of the differential interferogram has been deliberately minimized. A stored phase based on the sum channel may be adequate, or other approaches may be required. ${ }^{25}$ The approach to phase correction described below works well for the supercontinuum source built from our own stabilized fiber laser.

Before acquiring balanced interferograms, the relative gain and phase of the RF and DC channels are calibrated by recording simultaneous, low resolution, double sided interferograms on each channel with the signal beam blocked, thus exposing both acquisition channels to the same optical signal when $I_{\text {sig }}(x)=0$ :

$$
\begin{aligned}
& I_{D C}(x)=I_{\text {ref }}(x)+I_{\text {sig }}(x) \\
& I_{R F}(x)=A_{R F / D C}\left[I_{r e f}(x)-I_{\text {sig }}(x)\right]
\end{aligned}
$$

where $A_{R F / D C}$ is the relative gain of the RF channel compared to the DC channel. Applying a Forman phase correction ${ }^{26}$ and inverse Fourier transform to the phasecorrected reference-beam-only DC and RF interferograms yields a spectrum of the filtered supercontinuum and a phase spectrum for each channel. The scan-to-scan reproducibility in the spectra is typically within about $1 \%$, reflecting spectral variation as well as intensity fluctuations in the supercontinuum. The phase spectra $\varphi_{D C}(\sigma, r e f)$ and 
$\varphi_{R F}(\sigma, r e f)$ also display scan-to-scan variations, but the phase difference between the two channels $\Delta \varphi_{R F-D C}(\sigma, r e f) \equiv \varphi_{R F}(\sigma, r e f)-\varphi_{D C}(\sigma, r e f)$ is very reproducible, characterizing primarily a difference in the electronic time lag between the two detection channels. Similarly, the relative gain for the two channels, $A_{R F / D C}$, is a fixed property of the electronics and, like $\Delta \varphi_{R F-D C}(\sigma, r e f)$, can be measured once and saved for use in subsequent processing of dual beam differential interferograms.

The signal processing steps applied to the balanced sum and difference interferograms, $I_{D C}(x)$ and $I_{R F}(x)$ can be summarized as follows:

1. Apply Forman phase correction ${ }^{26}$ to the DC channel interferogram $I_{D C}(x)$ to generate a low resolution phase spectrum $\varphi_{D C}(\sigma)$ and a symmetrized interferogram $I_{D C}(x, s y m)$ at full, one-sided resolution.

2. Add the DC channel phase spectrum $\varphi_{D C}(\sigma)$ to the stored phase difference spectrum $\Delta \varphi_{R F-D C}(\sigma)$ to construct a total estimated phase, which is then used for a Forman phase correction of the RF channel interferogram, yielding $I_{R F}(x, s y m)$.

3. After correcting for the gain mismatch, $A_{R F / D C}$, in the two channels, the difference and sum of the symmetric RF and DC interferograms yields the signal and reference beam interferograms, algebraically rearranging equations (1)

$$
\begin{aligned}
& I_{\mathrm{Sig}}(x, \text { sym })=\frac{1}{2}\left[I_{D C}(x, \text { sym })-\frac{I_{R F}(x, s y m)}{A_{R F / D C}}\right] \\
& I_{\mathrm{Ref}}(x, \text { sym })=\frac{1}{2}\left[I_{D C}(x, \text { sym })+\frac{I_{R F}(x, s y m)}{A_{R F / D C}}\right]
\end{aligned}
$$


4. Finally, inverse real Fourier transforms of the symmetric signal and reference interferograms produce signal and reference spectra whose logarithmic ratio gives the absorbance, with good cancellation of source fluctuations, since most of the power in the calculated signal and reference interferograms has come from the same DC channel interferogram.

\section{RESULTS AND DISCUSSION}

To illustrate the limitations of single-beam methods with a supercontinuum source, we recorded a series of single-sweep interferograms with an evacuated sample cell and the same $9.5 \mathrm{~cm}$ cell with 0.04 Torr of a 50:50 mixture of $\mathrm{C}_{2} \mathrm{H}_{2}$ and $\mathrm{C}_{2} \mathrm{HD}$ to give a weak calibration signal in the $6200-6700 \mathrm{~cm}^{-1}$ region of interest. A single-beam spectrum of the bandpass filtered source is illustrated in Figure 2a, which shows the typical spectral structure the supercontinuum on a linear intensity scale. Relative to a stored background spectrum with an evacuated cell, the absorption spectrum in Figure $2 \mathrm{~b}$ shows structure from fluctuations in the background supercontinuum in addition to the weak acetylene line spectrum. Absorption features smaller than the background spectral fluctuations will be hard to measure, particularly if they are broad features rather than sharp lines.

Figure 3 illustrates the advantages of the present differential method, as implemented on a single photodetector. Figure $3 \mathrm{a}$ is the absorption spectrum acquired in a single mirror scan using the signal processing algorithm described above and the same sample used for the comparison single beam spectra of Figure 2. The curved baseline in Figure $3 \mathrm{a}$ is reproducible, and observed as well with an evacuated sample cell, and is the result of small chromatic mismatch between the signal and reference arms in the absence 
of an absorbing sample. If an empty-cell background spectrum is stored and used as a static reference, the final spectrum shown in Figure $3 b$ and expanded in Figure $3 c$ is obtained from a single sweep at a resolution of $0.1 \mathrm{~cm}^{-1}$, to be compared with the single channel spectrum of Figure $2 \mathrm{~b}$ and $2 \mathrm{c}$.

Compared to our previous measurements with signal and reference beams illuminating different detectors, with algebraic combinations of reference interferogram and the balanced output of the matched detectors, ${ }^{21}$ we find the present single-detector method to give comparable common-mode noise rejection of about $-40 \mathrm{~dB}$ with a simpler alignment and more stable operation. Additional tests were performed with a commercial supercontinuum source without the precise temperature control and low-noise current driver used in our home-built source. ${ }^{21}$ A similar $-40 \mathrm{~dB}$ common mode noise cancellation was attained with the noisier supercontinuum source, but even so, uncorrected source fluctuations would dominate the system performance for weak signals.

We expect that this single-detector balanced detection method may prove useful for application to longer wavelengths in the mid-infrared, where matched detector pairs less commonly available. (Note, however, the effective use ${ }^{18}$ of balanced $\mathrm{HgCdZnTe}$ detectors with a home-built autobalancing circuit based on the design of Hobbs). ${ }^{19}$ The bandwidth of the detector will need to extend up to the repetition rate of the supercontinuum in order to be useful. Sufficiently achromatic fiber collimation and beam splitting optics would also need to be incorporated into the optical design. Applications in attenuated total internal reflection or infrared reflection-absorption spectroscopy may be of particular interest, as these intrinsically weak absorption signals are not easily 
enhanced by massive multipass pathlength extension, and are not typically sharp spectra. For these types of applications, the suppression of baseline noise and fluctuation is crucial. The modest spectral resolution required for surface reflection spectroscopy is also well matched to this approach, since the scanning path difference of the interferometer must not approach the time delay between interleaved signal and reference beams. High resolution spectroscopy of gas phase samples is in any case better suited for cavity or aggressive multipass methods of signal enhancement, in contrast to the present emphasis on baseline noise and drift reduction.

\section{CONCLUSIONS}

A general method is described and demonstrated for reduction of fluctuation noise in FTS using a supercontinuum source. The time structure of the broadband optical source allows temporal interleaving of signal and reference beams onto a single photodetector, thereby encoding the differential interferogram at the RF frequency of the supercontinuum repetition rate, while the average interferogram appears at the audio frequency fringe rate of the scanning interferometer. After appropriate phase correction, the sum and difference of the average and differential interferograms yields signal and reference interferograms, from which weak absorption spectra can be calculated with strong suppression of common-mode noise. This implementation of balanced detection has been demonstrated for weak band spectra in the $1500-1600 \mathrm{~nm}$ region. 


\section{ACKNOWLEDGEMENTS}

This work was performed at Brookhaven National Laboratory under Contract No. DE-AC0298CH10886 and DE-SC0012704 with the U.S. Department of Energy and supported by its Division of Chemical Sciences, Geosciences, \& Biosciences, Office of Basic Energy Sciences. Additional support from the Goldhaber Distinguished Fellowship Program at BNL is gratefully acknowledged. We are grateful to I. Coddington for early assistance with design and construction of mode-locked fiber lasers. C.P. McRaven and T. J. Sears are acknowledged for

helpful discussions, and we thank M. G. White for shared access to the Bruker Vertex 80V used in this work. 


\section{FIGURE LEGENDS}

Figure 1. Schematic layout for differential FT spectrometry on a single detector. A supercontinuum source can be substituted for a conventional lamp source by inserting mirror (M1) into otherwise undisturbed beam path. After modulation in the commercial interferometer, the supercontinuum beam passes through bandpass filters (BP) clean-up polarizer $(\mathrm{P} 1)$, half-wave plate $(\lambda / 2)$, balancing polarizer $(\mathrm{P} 2)$ and polarizing beam splitter (BS1). The signal beam through the sample is combined with a reference beam, delayed by $50 \%$ of the supercontinuum repetition period, on polarizing beam combiner (BS2). The photodiode (D1) output is split into DC $(<20 \mathrm{kHz})$ and $\mathrm{RF}(>20 \mathrm{kHz})$ components and amplified. Phase-sensitive detection of the RF channel at the pulse repetition rate of the supercontinuum source (from D2) generates a difference interferogram, digitized in the spectrometer's 24 bit ADC, synchronously with another ADC channel recording the sum interferogram.

Figure 2. Single-beam supercontinuum FT spectra. (a) Spectral output of supercontinuum in the $6200-6700 \mathrm{~cm}^{-1}$ region. (b) Acetylene spectrum $(9.5 \mathrm{~cm}$ pathlength, 0.04 Torr $\mathrm{C}_{2} \mathrm{H}_{2} / \mathrm{C}_{2} \mathrm{HD}$ ) computed with stored background spectrum, demonstrating sweep-to-sweep variation in supercontinuum source. (c) Magnified region of the $v_{1}+v_{3} \mathrm{C}_{2} \mathrm{H}_{2}$ band and the $2 v_{1}$ band of $\mathrm{C}_{2} \mathrm{HD}$ from spectrum (b).

Figure 3. Differential supercontinuum FT spectra. (a) Single sweep absorption spectrum computed from simultaneously acquired DC and RF channels from a single photodetector 
(b) The same spectrum, normalized to a stored blank differential scan. (c) Magnified region of the $v_{1}+v_{3} \mathrm{C}_{2} \mathrm{H}_{2}$ band and the $2 v_{1}$ band of $\mathrm{C}_{2} \mathrm{HD}$ from spectrum (b). 


\section{FIGURES}

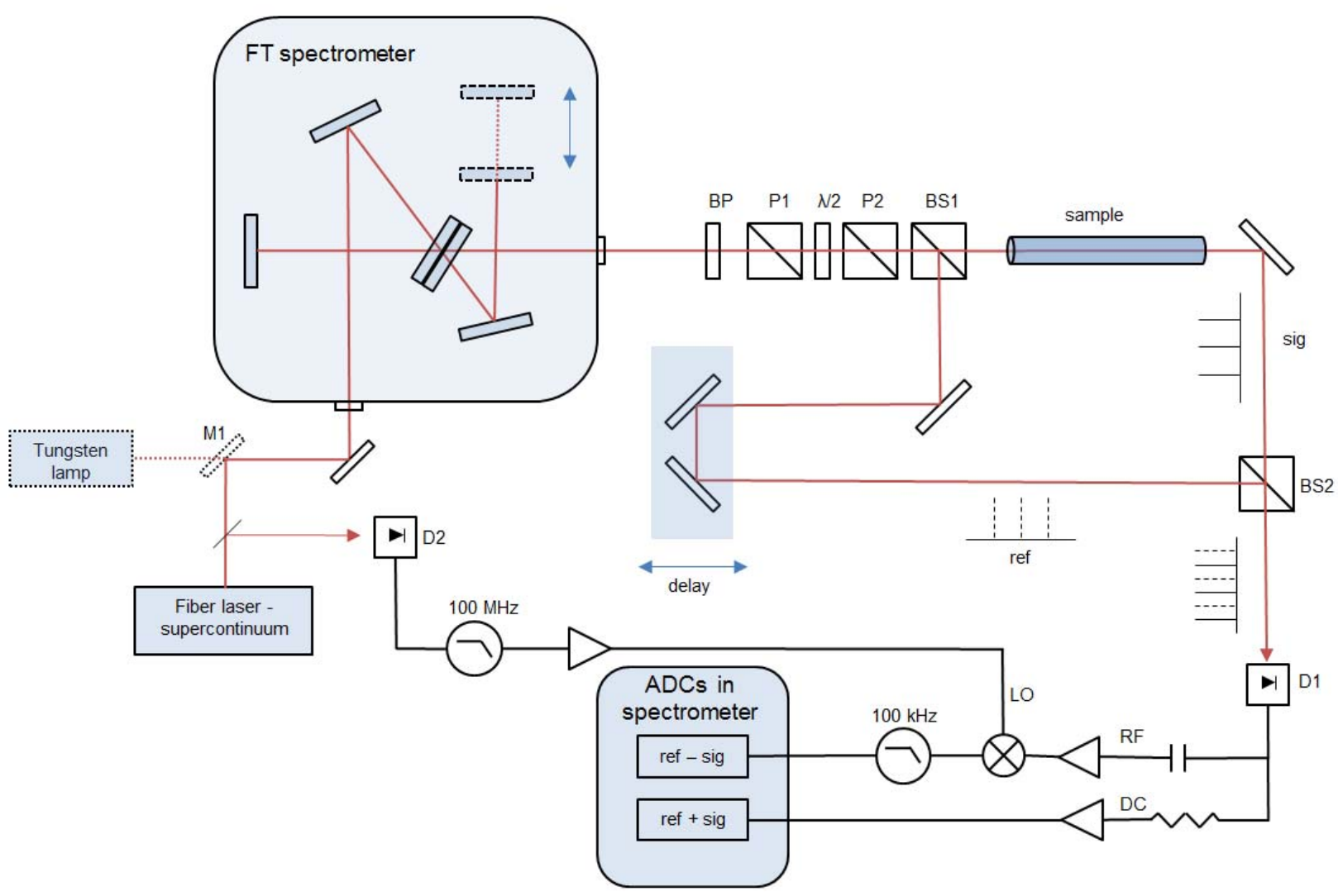

3 Goncharov \& Hall Figure 1.

Supercontinuum FTIR with Balanced Detection on a Single Photodiode 

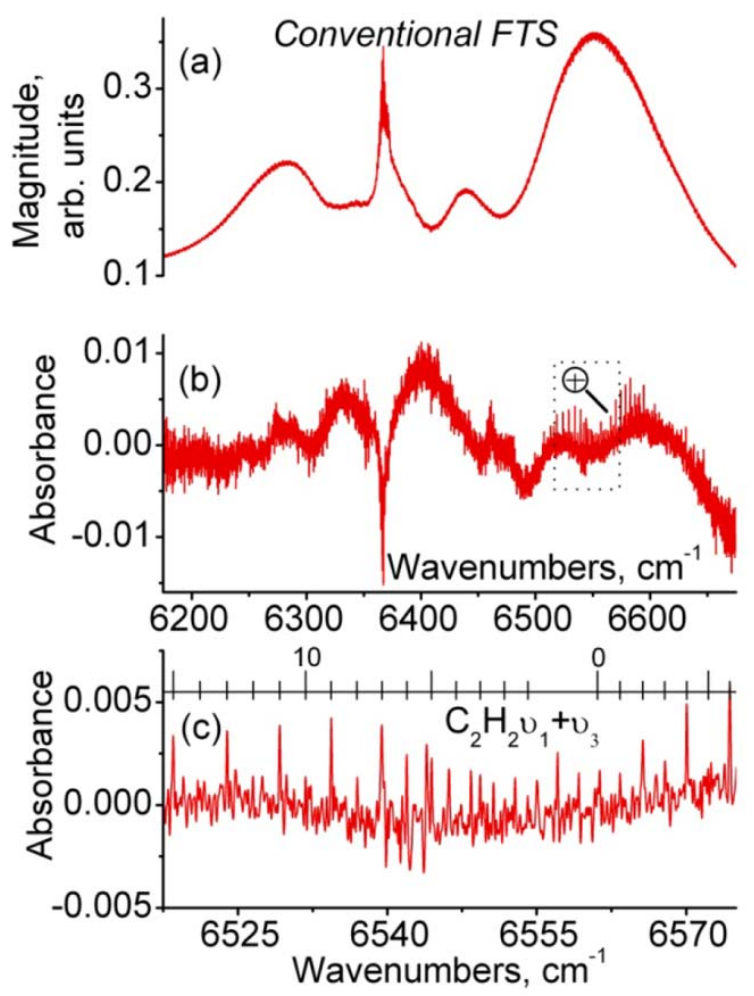

2 Goncharov \& Hall Figure 2. 

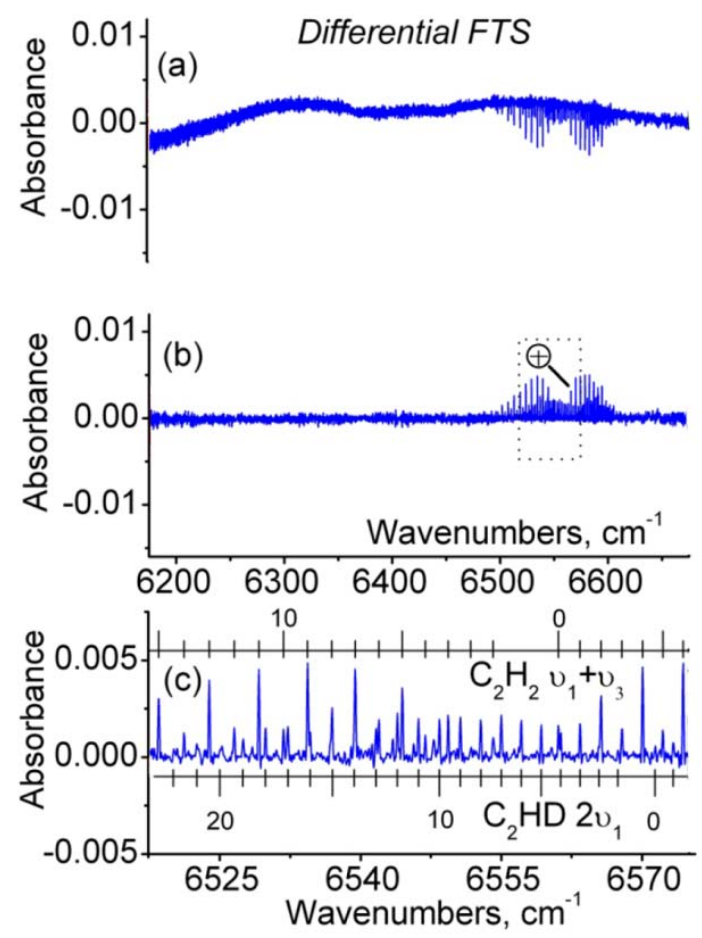

2 Goncharov \& Hall Figure 3. 


\section{REFERENCES}

[1] J. M. Dudley, G. Genty, and S. Coen, Rev. Mod. Phys. 78, 1135 (2006).

[2] J. M. Dunsby, and J. R. Taylor, Supercontinuum Generation in Optical Fibers (Cambridge University Press, Cambridge, 2010).

[3] J. Dudley, and G. Genty, Physics Today 66(7), 29 (2013).

[4] J. Mandon, G. Guelachvili, and N. Picque, Nat. Photon. 3, 99 (2009).

[5] W. Denzer, G. Hancock, M. Islam, C. E. Langley, R. Peverall, G. A. D. Ritchie, and D. Taylor, Analyst 136, 801 (2011).

[6] C. A. Michaels, T. Masiello, and P. M. Chu, Appl. Spectrosc. 63, 538 (2009).

[7] K. L. Corwin, N. R. Newbury, J. M. Dudley, S. Coen, S. A. Diddams, K. Weber, and R. S. Windeler, Phys. Rev. Lett. 90, 113904 (2003).

[8] T. Hirschfeld, Appl. Spectrosc. 30, 234 (1976).

[9] S. P. Davis, M. C. Abrams, and J. W. Brault, Fourier Transform Spectrometry (Academic Press, 2001).

[10] P. C. D. Hobbs, Building Electro-Optical Systems: Making It All Work (John Wiley \& Sons, Inc., Hoboken, NJ, 2009), 2nd edn.

[11] H. Bar-Lev, Infrared Physics 7, 93 (1967).

[12] D. Kuehl, and P. R. Griffiths, Anal. Chem. 50, 418 (1978).

[13] G. J. Kemeny, and P. R. Griffiths, Appl. Spectrosc. 34, 95 (1980).

[14] D. W. T. Griffith, and B. Galle, Atmos. Environ. 34, 1087 (2000).

[15] H. V. Andersen, A. Friderichsen, S. Clausen, and J. Bak, Appl. Opt. 44, 6167 (2005).

[16] P. R. Griffiths, and J. A. de Haseth, Fourier Transform Infrared Spectrometry, Second Ed., in Chemical Analysis (John Wiley \& Sons, Hoboken, NJ, 2006).

Supercontinuum FTIR with Balanced Detection on a Single Photodiode 
1 [17] A. Foltynwicz, T. Ban, P. Maslowski, F. Adler, and J. Ye, Phys. Rev. Lett. 107, 233002 2 (2011).

3 [18] A. Foltynwicz, P. Maslowski, A. J. Fleisher, B. J. Bjork, and J. Ye, Appl. Phys. B 110, 163 4 (2013).

5 [19] P. C. D. Hobbs, Appl. Opt. 36, 903 (1997).

6 [20] E. C. Robinson, J. Trägårdh, I. D. Lindsay, and H. Gersen, Rev. Sci. Instrum. 83, 063705 7 (2012).

8 [21] V. V. Goncharov, and G. E. Hall, Opt. Lett. 37, 2406 (2012).

9 [22] T. Buffeteau, B. Desbat, and J. M. Turlet, Appl. Spectrosc. 45, 380 (1991).

10 [23] M. J. Green, B. J. Barner, and R. M. Corn, Rev. Sci. Instrum. 62, 1426 (1991).

11 [24] T. Buffeteau, F. Lagugne-Labarthet, and C. Sourisseau, Appl. Spectrosc. 59, 732 (2005).

12 [25] M. S. Huston, and M. S. Braiman, Appl. Spectrosc. 52, 974 (1998).

13 [26] M. L. Forman, W. H. Steel, and G. A. Vanasse, J. Opt. Soc. Am. 56, 59 (1966).

14

15 